\title{
Hammerstein integral equation with a generalized singular kernel
}

\author{
A. M. AL-Bugami \\ Department of Mathematics, Faculty of Sciences, Taif University, Saudi Arabia. \\ E-mail:abeer101aa@yahoo.com
}

\begin{abstract}
In this work, the Hammerstein integral equation (HIE), with a generalized singular kernel, is considered and solved numerically, using Product Trapezoidal rule, Toeplitz matrix method and Product Nystrom method. The existence and uniqueness solution, under certain conditions, are considered. Moreover, numerical results when the kernel takes a generalized logarithmic form, Carleman function and Cauchy kernel are investigated. Also the error, in each case, is estimated.
\end{abstract}

Keywords: Hammerstein singular integral equation, Product Trapezoidal rule, Toeplitz matrix, product Nystrom method, generalized logarithmic form, generalized Carleman function.

\section{Introduction}

The integral equations of various types and kinds play an important role in many branches of mathematics. Many problems of fracture mechanics, aerodynamics, the theory of porous filtering, antenna problems in electromagnetic theory and others can be formulated as integral equations of the first, second and third kind. The solutions of their applications can be obtained analytically, using the theory developed by Muskhelishvili [1]. At the same time the sense of numerical methods takes an important place in solving integral equations.

Many methods are used to obtain the solution of the nonlinear Fredholm integral equation. In [2], Abdou et al., obtained numerically the solution of the singular Fredholm integral equation. Abdou et. al., in [3], obtained a numerical solution for the nonlinear integral equation of Hammerstein type. In [4], Abdou and Hendi, solved the Fredholm integral equation with Hilbert kernel numerically. In [5], Abdou obtained the solution of linear and nonlinear integral equation. In [6], Abdou and AL-Bugami studied Fredholm integral equation with a generalized singular kernel in the linear case and solved this equation numerically. Emamzadeh and Kajani in [7] solved nonlinear Fredholm integral equation by using quadrature Methods. In [8], Ahmad and Akbar solved nonlinear Fredholm integral equation using Lagrange Functions.

In this paper, we consider the Hammerstein integral equation with a generalized singular kernel of the form

$$
\mu \phi(t)-\lambda \int_{-a}^{a} k(|g(t)-g(\zeta)|) \gamma(t, \zeta, \phi(\zeta)) d \zeta=f(t)
$$

where the free term $f(t)$ and the kernel $k(|g(t)-g(\zeta)|)$ are known functions, and $\phi(t)$ is the unknown function to be determined. The numerical coefficient $\lambda$ is called the parameter of the integral equation, may be complex, and has many physical meaning. While the parameter $\mu$ defines the kind of the integral equation. The function $g(t)$ is a continuous with its derivatives in $t \in[-a, a]$.

\section{The existence and uniqueness solution}

In this section, the existence and uniqueness solution of Eq. (1), under certain conditions, will be discussed and proved using Banach fixed point theorem. For this, we state the following theorem: 


\section{Theorem 1: (Banach Fixed Point Theorem, see Kreysig [9])}

Consider a metric space $X=(X, d)$, where $X \neq \Phi$. Suppose that $X$ is complete and let $T: X \rightarrow X$ be a contraction on $X$. Then $T$ has precisely one fixed point.

In the light of Theorem 1, we write Eq. (1) in the integral operator form

$$
\bar{W} \phi(t)=\frac{1}{\mu} f(t)+W \phi(t), \quad(\mu \neq 0)
$$

Where,

$$
W \phi(t)=\frac{\lambda}{\mu} \int_{-a}^{a} k(|g(t)-g(\zeta)|) \gamma(t, \zeta, \phi(\zeta)) d \zeta
$$

Also, we assume the following conditions:

(i) The kernel $k(|g(t)-g(\zeta)|)$ satisfies the discontinuity condition

$$
\left\{\int_{-a-a}^{a} \int_{a}^{a}|k(|g(t)-g(\zeta)|)|^{2} d t d \zeta\right\}^{\frac{1}{2}}=c<\infty \quad,(c \text { is a constant }) \text {. }
$$

(ii) The given function $f(t)$ is continuous in the space $L_{2}[-a, a]$, and its norm is defined as $\|f(t)\|_{L_{2}[-a, a]}=\left\{\int_{-a}^{a}|f(t)|^{2} d t\right\}^{\frac{1}{2}}=N, \quad(N$ is a constant $)$.

(iii) The known continuous function $\gamma(t, \zeta, \phi(t))$ satisfies, for the constants $A>A_{1}, A>P$, the following conditions

(a) $\left\{\int_{-a}^{a}|\gamma(t, \zeta, \phi(t))|^{2} d t\right\}^{\frac{1}{2}} \leq A_{1}\|\phi(t)\|_{L_{2}[-a, a]}$,

(b) $\left|\gamma\left(t, \zeta, \phi_{1}(t)\right)-\gamma\left(t, \zeta, \phi_{2}(t)\right)\right| \leq M(t)\left|\phi_{1}(t)-\phi_{2}(t)\right|$, where $\|M(t)\|_{L_{2}[-a, a]}=P$.

\section{Theorem 2:}

If the conditions (i)-(iii) are verified, then Eq. (1) has a unique solution in the Banach space $L_{2}[-a, a]$. The proof of this theorem depends on the following two lemmas:

\section{Lemma 1:}

Under the conditions (i)-(iii-a), the operator $\bar{W}$ defined by (2), maps the space $L_{2}[-a, a]$ into itself.

\section{Proof:}

From the formulas (2) and (3), we get

$$
\|\bar{W} \phi(t)\|_{L_{2}[-a, a]} \leq \frac{1}{|\mu|}\|f(t)\|_{L_{2}[-a, a]}+\left|\frac{\lambda}{\mu}\right||| \int_{-a}^{a}|k(|g(t)-g(\zeta)|)||\gamma(t, \zeta, \phi(\zeta))| d \zeta \|_{L_{2}[-a, a]}
$$

Using the condition (ii), then applying Cauchy-Schwarz inequality, we have

$$
\|\bar{W} \phi(t)\|_{L_{2}[-a, a]} \leq \frac{N}{|\mu|}+\left|\frac{\lambda}{\mu}\right|\left\{\int_{-a-a}^{a} \int_{-a}^{a}|k(|g(t)-g(\zeta)|)|^{2} d t d \zeta\right\}^{\frac{1}{2}}\left\{\int_{-a}^{a}|\gamma(t, \zeta, \phi(\zeta))|^{2} d \zeta\right\}^{\frac{1}{2}} \text {. }
$$

In the light of the conditions (i) and (iii-a), the above inequality takes the form

$$
\|\bar{W} \phi(t)\|_{L_{2}[-a, a]} \leq \frac{N}{|\mu|}+\sigma\|\phi(t)\|_{L_{2}[-a, a]},\left(\sigma=\left|\frac{\lambda}{\mu}\right| c A\right) .
$$


The last inequality (4) shows that, the operator $\bar{W}$ maps the ball $S_{r}$ into itself, where

$$
r=\frac{N}{(|\mu|-|\lambda| c A)}
$$

Since $r>0, N>0$, therefore we have $\sigma<1$. Moreover, the inequality (4) involves the boundedness of the operator $W$ of Eq. (2), where

$$
\|W \phi(t)\|_{L_{2}[-a, a]} \leq \sigma\|\phi(t)\|_{L_{2}[-a, a]} .
$$

Also, the inequalities (4) and (6) define the boundedness of the operator $\bar{W}$.

\section{Lemma 2 :}

If the two conditions (i) and (iii-b) are satisfied, then the operator $\bar{W}$ is contractive in the Banach space $L_{2}[-a, a]$.

\section{Proof:}

For the two functions $\phi_{1}(t)$ and $\phi_{2}(t)$ in the space $L_{2}[-a, a]$, the formulas (2), (3) lead to $\left\|\left(\bar{W} \phi_{1}-\bar{W} \phi_{2}\right)(t)\right\|_{L_{2}[-a, a]} \leq\left|\frac{\lambda}{\mu}\right|\left\|\int_{-a}^{a}|k(|g(t)-g(\zeta)|)|\left|\gamma\left(t, \zeta, \phi_{1}(\zeta)\right)-\gamma\left(t, \zeta, \phi_{2}(\zeta)\right)\right| d \zeta\right\|_{L_{2}[-a, a]}$

Using the condition (iii-b), then applying Cauchy-Schwarz inequality, we have

$$
\left\|\left(\bar{W} \phi_{1}-\bar{W} \phi_{2}\right)(t)\right\|_{L_{2}[-a, a]} \leq\left|\frac{\lambda}{\mu}\right|\left(\int_{-a-a}^{a} \int_{-a}^{a}|k(|g(t)-g(\zeta)|)|^{2} d t d \zeta\right)^{\frac{1}{2}}\left(\int_{-a}^{a} M^{2}(\zeta)\left|\phi_{1}(\zeta)-\phi_{2}(\zeta)\right|^{2} d \zeta\right)^{\frac{1}{2}}
$$

Finally, with the aid of conditions (i) and (iii-b), we obtain

$$
\left\|\left(W \bar{W} \phi_{1}-\bar{W} \phi_{2}\right)(t)\right\|_{L_{2}[-a, a]} \leq \sigma\left\|\phi_{1}(t)-\phi_{2}(t)\right\|_{L_{2}[-a, a]} .
$$

Inequality (7) shows that, the operator $\bar{W}$ is continuous in the space $L_{2}[-a, a]$, then $\bar{W}$ is a contraction operator under the condition $\sigma<1$. The proof of Theorem 2 is directly obtained after the following discussion: Since the previous two lemmas showed that the operator $\bar{W}$ is contractive in the Banach space $L_{2}[-a, a]$, then by Theorem 1 , the operator $\bar{W}$ has a unique fixed point which is, of course, the unique solution of Eq. (1).

\section{Some different numerical methods}

In this section, we discuss the solution of Hammerstein integral equation with a generalized singular kernel using three different methods and determine the error in each one.

\subsection{Product trapezoidal rule of HIE with a generalized singular kernel}

The product trapezoidal rule is constructed after approximating $\gamma(t, \zeta, \phi(\zeta))$ by piecewise nonlinear functions, in particular

$$
\begin{aligned}
& \gamma(t, \zeta, \phi(t)) \approx \frac{\zeta-t_{k}}{h} \gamma\left(t, t_{k+1}, \phi\left(t_{k+1}\right)\right)+\frac{t_{k+1}-\zeta}{h} \gamma\left(t, t_{k}, \phi\left(t_{k}\right)\right), \\
& h=t_{k+1}-t_{k}, t_{k} \leq \zeta \leq t_{k+1} .
\end{aligned}
$$

This leads to the following : 


$$
\begin{aligned}
\int_{-a_{i}}^{a_{i}} k\left(\left|g\left(t_{i}\right)-g(\zeta)\right|\right) \gamma\left(t_{i}, \zeta, \phi(\zeta)\right) d \zeta & =\alpha_{i 1} \gamma\left(t_{i}, t_{0}, \phi\left(t_{0}\right)\right) \\
& +\sum_{k=1}^{i-1}\left(\alpha_{i, k+1}+\beta_{i k}\right) \gamma\left(t_{i}, t_{k}, \phi\left(t_{k}\right)\right)+\beta_{i i} \gamma\left(t_{i}, t_{i}, \phi\left(t_{i}\right)\right)+R_{i}^{(p)},
\end{aligned}
$$

where

$$
\begin{aligned}
& \alpha_{i, k+1}=\frac{1}{h} \int_{a_{k}}^{a_{k+1}}\left(t_{k+1}-\zeta\right) k\left(\left|g\left(t_{i}\right)-g(\zeta)\right|\right) d \zeta \\
& \beta_{i, k+1}=\frac{1}{h} \int_{a_{k}}^{a_{k+1}}\left(\zeta-t_{k}\right) k\left(\left|g\left(t_{i}\right)-g(\zeta)\right|\right) d \zeta .
\end{aligned}
$$

The numerical solution $\phi_{i}$ of Eq. (1) is

With

$$
\begin{gathered}
\phi_{i}=\frac{1}{\mu} f\left(t_{i}\right)+\frac{\lambda}{\mu}\left[\alpha_{i 1} \gamma\left(t_{i}, t_{0}, \phi\left(t_{0}\right)\right)+\sum_{k=1}^{i-1}\left(\alpha_{i, k+1}, \beta_{i k}\right) \gamma\left(t_{i}, t_{k}, \phi\left(t_{k}\right)\right)\right. \\
\left.+\beta_{i i} \gamma\left(t_{i}, t_{i}, \phi\left(t_{i}\right)\right)\right], \quad i=1,2,3, \ldots,
\end{gathered}
$$

$$
\mu \phi_{0}=f\left(t_{0}\right)
$$

Using Newton-Raphson method, the value of $\phi_{i}$ can be determining $\phi_{1}, \phi_{2}, \ldots$

The error of the product trapezoidal is defined by

$$
\begin{aligned}
R_{i}^{(p)}=\mid \int_{-a_{i}}^{a_{i}} k\left(\mid g\left(t_{i}\right)-\right. & g(\zeta) \mid) \gamma\left(t_{i}, \zeta, \phi(\zeta)\right) d \zeta-\left[\alpha_{i 1} \gamma\left(t_{i}, t_{0}, \phi\left(t_{0}\right)\right)\right. \\
& \left.+\sum_{k=1}^{i-1}\left(\alpha_{i, k+1}+\beta_{i k}\right) \gamma\left(t_{i}, t_{k}, \phi\left(t_{k}\right)\right)+\beta_{i i} \gamma\left(t_{i}, t_{i}, \phi\left(t_{i}\right)\right)\right] \mid .
\end{aligned}
$$

\subsection{The toeplitz matrix method of HIE with a generalized singular kernel}

To discuss the solution of Eq. (1) numerically, using Toeplitz matrix method, we write the integral term as:

$$
\int_{-a}^{a} k\left(|g(t)-g(\zeta)| \gamma(t, \zeta, \phi(\zeta)) d \zeta=\sum_{n=-N}^{N} \int_{n h}^{n h+h} k(|g(t)-g(\zeta)|) \gamma(t, \zeta, \phi(\zeta)) d \zeta \quad,\left(h=\frac{a}{N}\right)\right.
$$

Then, we approximate the integral term in the right hand side by

$$
\int_{n h}^{n h+h} k(|g(t)-g(\zeta)|) \gamma(t, \zeta, \phi(\zeta)) d \zeta=A_{n}(g(t)) \gamma(t, n h, \phi(n h))+B_{n}(g(t)) \gamma(t, n h+h, \phi(n h+h))+R
$$

where $A_{n}(g(t)), B_{n}(g(t))$ are two arbitrary functions to be determined and $R$ is the error estimate.

As the principle idea of the Toeplitz matrix to obtain the values of the function $A_{n}(g(t)), B_{n}(g(t))$, we assume $\gamma(t, \zeta, \phi(\zeta))=g^{\prime}(\zeta), g^{\prime}(\zeta) g(\zeta)$ respectively, in Eq. (15), where $g^{\prime}(t)$ is a monotonic increasing function. This yields a set of two equations in terms of two unknown functions where, in this case, the error is vanishing. Solving the results we have: $A_{n}(g(t))=\frac{1}{g^{\prime}(n h)(g(n h+h)-g(n h))}[g(n h+h) I(t)-J(t)]$, 


$$
B_{n}(g(t))=\frac{1}{g^{\prime}(n h+h)(g(n h+h)-g(n h)}[J(t)-g(n h) I(t)]
$$

where

$$
I(t)=\int_{n h}^{n h+h} k(|g(t)-g(\zeta)|) \cdot g^{\prime}(\zeta) d \zeta \quad, J(t)=\int_{n h}^{n h+h} k(|g(t)-g(\zeta)|) \cdot g^{\prime}(\zeta) \cdot g(\zeta) d \zeta
$$

Using (16) in (15), the integral equation (1) becomes

$$
\mu \phi(t)-\lambda \sum_{n=-N}^{N} D_{n}(g(t)) \phi(n h)=f(t)
$$

Using the following notation, for $t=m h$,

$$
\phi(t)=\phi(m h)=\phi_{m}, D_{n}(g(t))=D_{n}(g(m h))=D_{n, m}, f(t)=f(m h)=f_{m},
$$

we get the following system of linear algebraic equations

$$
\mu \phi_{m}-\lambda \sum_{n=-N}^{N} D_{n, m} \phi_{n}=f_{m}
$$

where

$$
D_{n, m}=\left\{\begin{array}{cl}
A_{-N}(g(m h)) & , n=-N \\
A_{n}(g(m h))+B_{n-1}(g(m h)) & , \quad-N<n<N \\
B_{N-1}(g(m h)) & , n=N
\end{array}\right.
$$

The matrix $D_{n, m}$ may be written as $D_{n, m}=G_{n, m}-E_{n, m}$, where

$$
G_{n, m}=A_{n}(g(m h))+B_{n-1}(g(m h)) \quad, \quad-N \leq m, n \leq N
$$

is a Toeplitz matrix of order $2 N+1$ and

$$
E_{n, m}=\left\{\begin{array}{cr}
B_{-N-1}(g(m h)) & , n=-N \\
0 & , N<n<N \\
A_{N}(g(m h)) & , n=N
\end{array}\right.
$$

represents a matrix of order $2 N+1$ whose elements are zeros except the first and the last columns (rows). The solution of the system (20) can be obtained in the form

$$
\phi(m h)=\left[\mu I-\lambda\left(G_{n, m}-E_{n, m}\right)\right]^{-1} f(m h) \quad, \quad\left|\mu I-\lambda\left(\mathrm{G}_{n, m}-E_{n, m}\right)\right| \neq 0
$$

The error term $R$ is determined from equation (15) by letting $\gamma(t, \zeta, \phi(\zeta))=g^{\prime} g^{2}$ to get,

$$
R=\left|\int_{n h}^{n h+h} g^{\prime} g^{2} k(|g(t)-g(\zeta)|) d \zeta-A_{n}(g(t)) g^{\prime}(n h) g^{2}(n h)-B_{n}(g(t)) g^{\prime}(n h+h) g^{2}(n h+h)\right|
$$


(1) The method is said to be convergent of order $r$ in $[-a, a]$ if and only if for $N$ sufficiently large there exists a constant $D>0$, independent of $N$, such that

$\left\|\phi(t)-\phi_{N}(t)\right\| \leq D N^{-r}$.

(2) The linear algebraic system (20) has a unique solution, under the convergence condition:

$$
\sup _{N}\left\|\sum_{n=-N}^{N} D_{n m}\right\| \leq c^{\prime} \quad\left(c^{\prime} \text { is a constant }\right)
$$

\subsection{The product Nystrom method of HIE with a generalized singular kernel}

We discuss the solution of Fredholm integral equation using the product Nystrom method.

Consider the integral equation

$$
\mu \phi(t)-\lambda \int_{-a}^{a} p(g(t), g(\zeta)) \bar{k}(|g(t)-g(\zeta)|) \gamma(t, \zeta, \phi(\zeta)) d \zeta=f(t)
$$

when the kernel $k(|g(t)-g(\zeta)|)$ is singular within the range of integration. We can often factor out the singularity in $k(|g(t)-g(\zeta)|)$ by writing it in the form

$$
k(|g(t)-g(\zeta)|)=p(|g(t)-g(\zeta)|) \bar{k}(|g(t)-g(\zeta)|)
$$

Where $p$ and $\bar{k}$ are respectively badly behaved and well behaved functions of their arguments, respectively, $\phi(t)$ is the unknown function, while $f(t)$ is a given function. Equation (26) can be written in the form

$$
\mu \phi\left(t_{i}\right)-\lambda \sum_{j=0}^{N} w_{i j} \bar{k}\left(\left|g\left(t_{i}\right)-g\left(\zeta_{j}\right)\right|\right) \gamma\left(t_{i}, t_{j}, \phi_{i}\left(\zeta_{j}\right)\right)=f\left(t_{i}\right) \quad, \quad i=0,1, \ldots, N
$$

where $t_{i}=\zeta_{i}=a+i h, i=0,1, \ldots, N$ with $h=\frac{2 a}{N}$ and $N$ even, and $w_{i j}$ are weights. As in Delves and Mohamed [10], we write:

$$
\begin{aligned}
& \int_{-a}^{a} p\left(g\left(t_{i}\right), g(\zeta)\right) \bar{k}\left(\left|g\left(t_{i}\right)-g(\zeta)\right|\right) \gamma(t, \zeta, \phi(\zeta)) d \zeta \approx \sum_{j=0}^{\frac{N-2}{2} y_{y_{2 j+2}}} p\left(g\left(t_{i}\right), g(\zeta)\right) \cdot\left\{\frac{\left(g\left(\zeta_{2 j+1}\right)-g(\zeta)\right)\left(g\left(\zeta_{2 j+2}\right)-g(\zeta)\right)}{g(h) g(2 h)}\right. \\
& \bar{k}\left(\left|g\left(t_{i}\right)-g\left(\zeta_{2 j}\right)\right|\right) \gamma\left(t_{i}, \zeta_{2 j}, \phi\left(\zeta_{2 j}\right)\right)+\frac{\left(g(\zeta)-g\left(\zeta_{2 j}\right)\right)\left(g\left(\zeta_{2 j+2}\right)-g(\zeta)\right)}{g(h) g(h)} \bar{k}\left(\left|g\left(t_{i}\right)-g\left(\zeta_{2 j+1}\right)\right|\right) \gamma\left(t_{i}, \zeta_{2 j+1}, \phi\left(\zeta_{2 j+1}\right)\right) \\
& \left.+\frac{\left(g(\zeta)-g\left(\zeta_{2 j+1}\right)\right)\left(g(\zeta)-g\left(\zeta_{2 j}\right)\right)}{g(2 h) g(h)} \bar{k}\left(\left|g\left(t_{i}\right)-g\left(\zeta_{2 j+2}\right)\right|\right) \gamma\left(t_{i}, \zeta_{2 j+2}, \phi\left(\zeta_{2 j+2}\right)\right)\right\} d \zeta \\
& =\sum_{j=0}^{N} w_{i j} \bar{k}\left(g\left(t_{i}\right)-g\left(\zeta_{j}\right) \mid\right) \gamma\left(t_{i}, \zeta_{j}, \phi\left(\zeta_{j}\right)\right)
\end{aligned}
$$

therefore:

$$
\begin{aligned}
& w_{i, 0}=\beta_{1}\left(\zeta_{i}\right) \quad, \quad w_{i, 2 j+1}=2 \gamma_{j+1}\left(\zeta_{i}\right) \\
& w_{i, 2 j}=\alpha_{j}\left(\zeta_{i}\right)+\beta_{j+1}\left(\zeta_{i}\right) \quad, \quad w_{i, N}=\alpha_{N / 2}\left(\zeta_{i}\right)
\end{aligned}
$$


where,

$$
\begin{aligned}
& \alpha_{i j}=\frac{(g(h))^{2}}{g(2 h)} \int_{0}^{2} \eta(\eta-1) p\left(g\left(\zeta_{2 j-2}+\eta h\right), g\left(\zeta_{i}\right)\right) d \eta \\
& \beta_{i j}=\frac{(g(h))^{2}}{g(2 h)} \int_{0}^{2}(\eta-1)(\eta-2) p\left(g\left(\zeta_{2 j-2}+\eta h\right), g\left(\zeta_{i}\right)\right) d \eta \\
& \gamma_{i j}=\frac{(g(h))^{2}}{g(2 h)} \int_{0}^{2} \eta(2-\eta) p\left(g\left(\zeta_{2 j-2}+\eta h\right), g\left(\zeta_{i}\right)\right) d \eta
\end{aligned}
$$

Therefore, the Here, in (31), we introduce the variable $g(\zeta)=g\left(\zeta_{2 j-2}\right)+\eta g(h), 0 \leq \eta \leq 2$. system (31) has a solution.

$$
\Phi=[\mu I-\lambda W]^{-1} F
$$

where $I$ is the identity matrix, and $|\mu I-\lambda W| \neq 0$

\section{Numerical results}

In this section, we apply the previous methods to obtain numerical results for Eq. (1), with logarithmic kernel, Carleman function and Cauchy kernel. By using Maple 10 the approximate solution and the error, in each case, is obtained and computed, respectively.

Example 1: For integral equation:

$$
\phi(t)-\lambda \int_{-1}^{1} \ln \left|t^{3}-\zeta^{3}\right| \phi^{2}(\zeta) d \zeta=f(t), \quad\left(\text { exact solution } \phi(t)=t^{5},|t| \leq 1\right)
$$

The product trapezoidal rule, Toeplitz matrix method and product Nystrom method are used to get the approximate solution for values of $\mu=1, \lambda=0.01,0.1$ and $N=41$ units.

Err. PTR

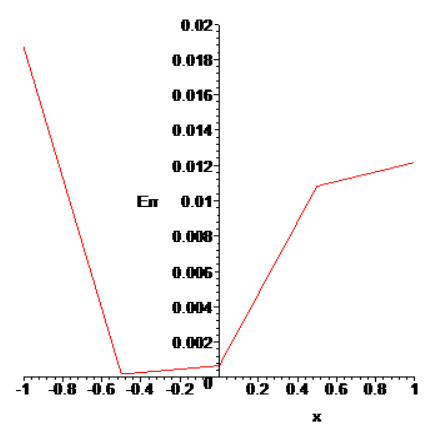

Err. TMM

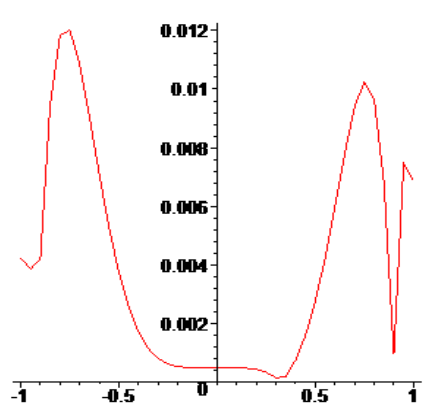

Err. PNM

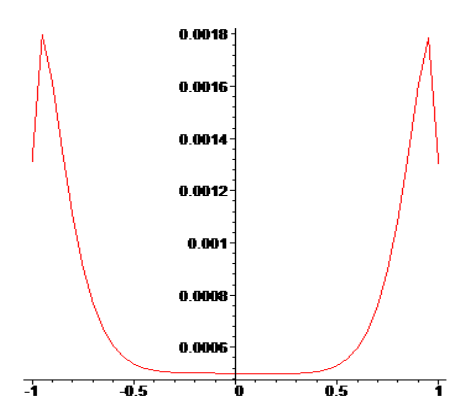

Fig. 1: The error values by using product trapezoidal rule, Toeplitz matrix method and product Nystrom method at $\lambda=0.01$ 
Err. PTR

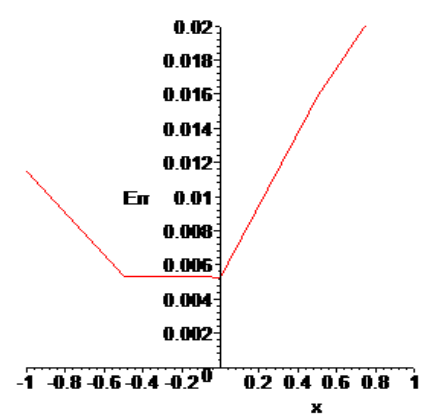

Err. TMM

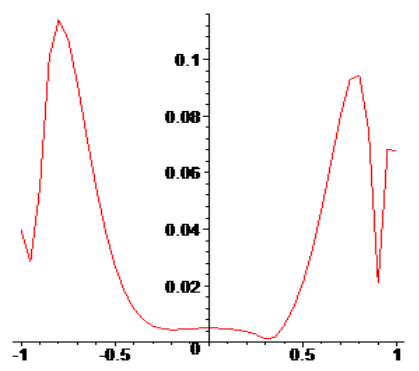

Err. PNM

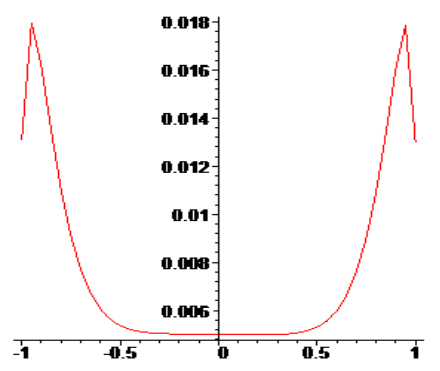

Fig. 2: The error values by using product trapezoidal rule, Toeplitz matrix method and product Nystrom method at $\lambda=0.1$

Example 2: For integral equation

$\phi(t)-\lambda \int_{-1}^{1} \ln \left|e^{t}-e^{\zeta}\right|(\phi(\zeta))^{2} d \zeta=f(t), \quad\left(\right.$ exact solution $\left.\phi(t)=e^{t}\right)$

The product trapezoidal rule, Toeplitz matrix method and product Nystrom method are used to get the approximate solution for values of $\mu=1, \lambda=0.001,0.01$, and $N=41$ units.

Err. PTR

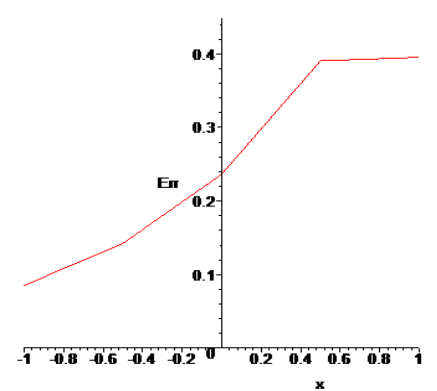

Err. TMM

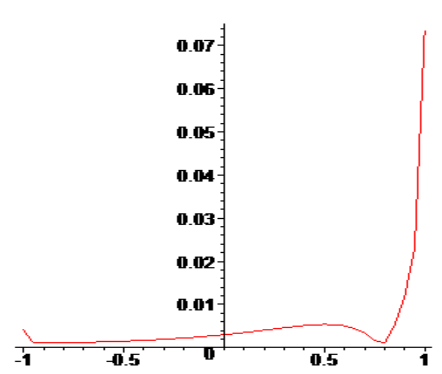

Err. PNM

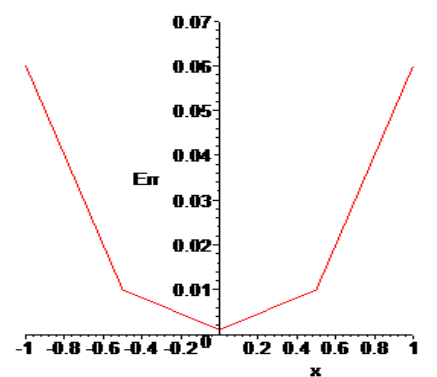

Fig. 3: The error values by using product trapezoidal rule, Toeplitz matrix method and product Nystrom method at $\lambda=0.001$

Err. PTR

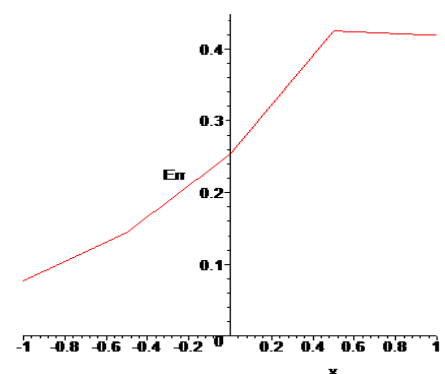

Err. TMM

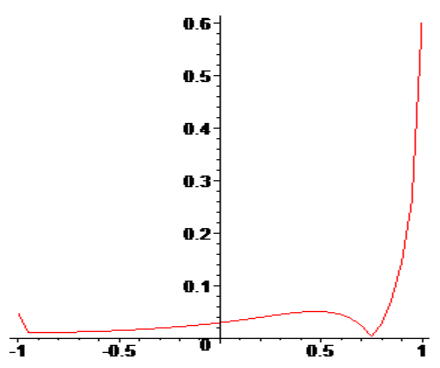

Err. PNM

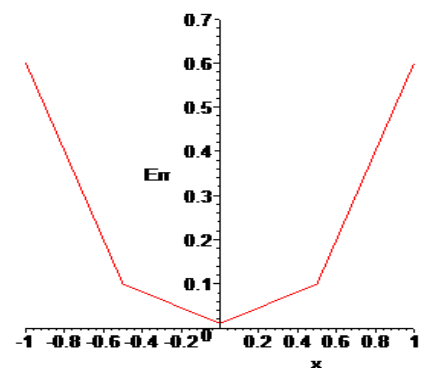

Fig. 4: The error values by using product trapezoidal rule, Toeplitz matrix method and product Nystrom method at $\lambda=0.01$

Example 3: Consider the integral equation 
$\phi(t)-\lambda \int_{-1}^{1}\left|t^{3}-\zeta^{3}\right|^{-v} \phi^{2}(\zeta) d \zeta=f(t), \quad\left(\right.$ exact solution $\left.\phi(t)=t^{5}\right)$

Here The product trapezoidal rule, Toeplitz matrix method and product Nystrom method are used to get the approximate solution for values of $\mu=1, \lambda=0.01, v=0.1,0.2$, and $N=41$ units.
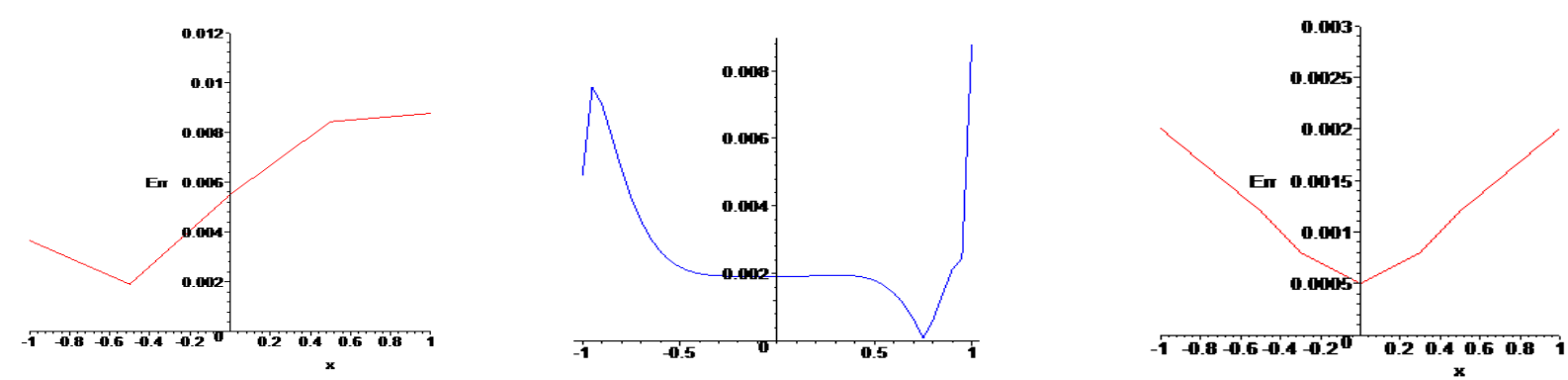

Fig. 5: The error values by using product trapezoidal rule, Toeplitz matrix method and product Nystrom method at $\lambda=0.01, v=0.1$

\section{Err. PTR}

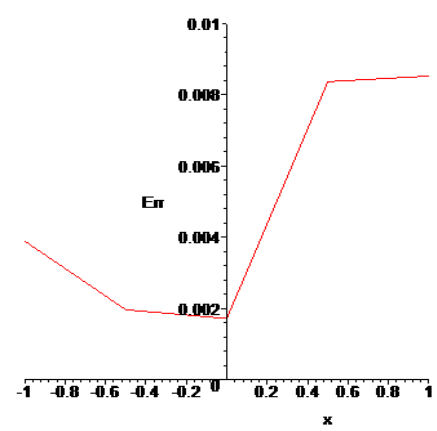

Err. TMM

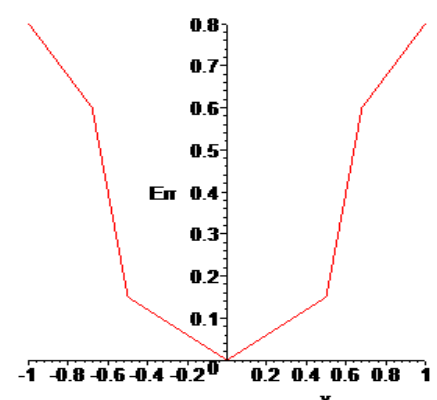

$\mathbf{x}$

\section{Err. PNM}

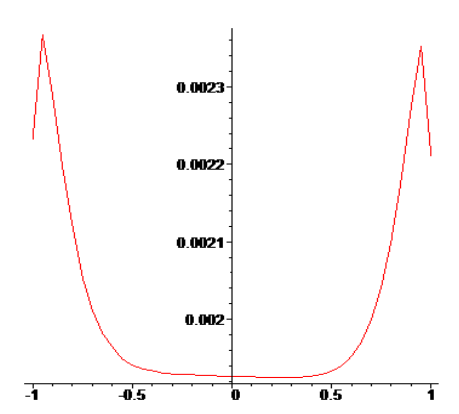

Fig. 6: The error values by using product trapezoidal rule, Toeplitz matrix method and product Nystrom method at $\lambda=0.01, v=0.2$

Example 4: Consider the integral equation

$\phi(t)-\lambda \int_{-1}^{1}|\sin (t)-\sin (\zeta)|^{-v} \phi^{2}(\zeta) d \zeta=f(t), \quad($ exact solution $\phi(t)=\cos (t))$

Here, the Toeplitz matrix method and product Nystrom method are used to get the approximate solution for values of $\mu$ $=1, \lambda=0.01, v=0.1,0.22$ and $N=41$ units. 
Err. PTR

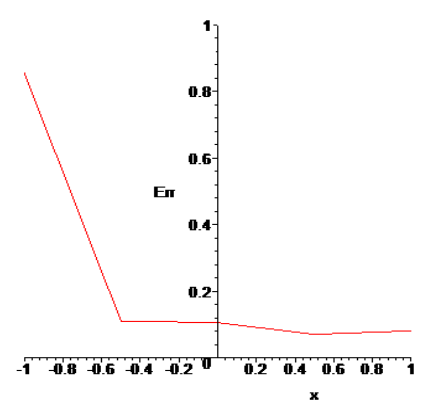

Err. TMM

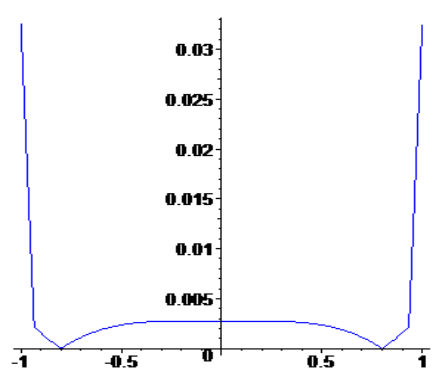

Err. PNM

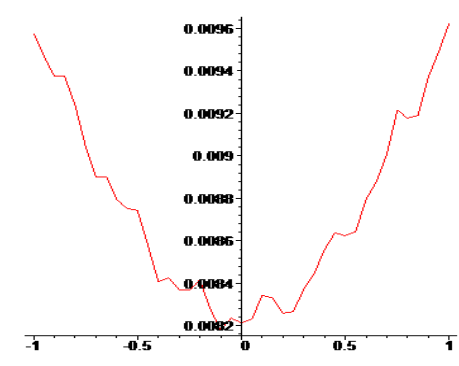

Fig. 7: The error values by using product trapezoidal rule, Toeplitz matrix method and product Nystrom method at $\lambda=0.01, v=0.1$

Err. PTR

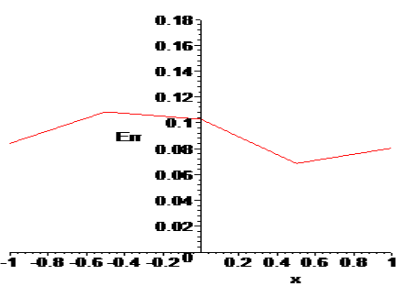

Err. TMM

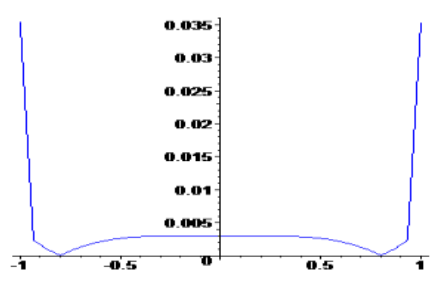

Err. PNM

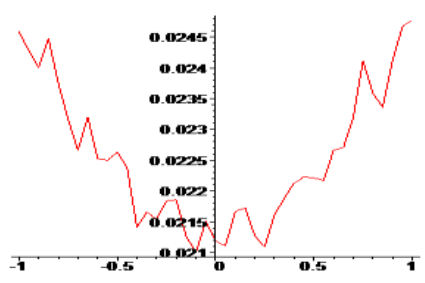

Fig. 8: The error values by using product trapezoidal rule, Toeplitz matrix method and product Nystrom method at $\lambda=0.01, v=0.22$

Example 5: Consider the integral equation

$\phi(t)-\lambda \int_{-1}^{1}\left(\frac{1}{t^{2}-\zeta^{2}}\right) \phi^{2}(\zeta) d \zeta=f(t), \quad\left(\right.$ exact solution $\left.\phi(t)=t^{5}\right)$

Here, the Toeplitz matrix method and product Nystrom method are used to get the approximate solution for values of $\mu$ $=1, \lambda=0.01,0.1$ and $N=41$ units.

\section{Err. PTR}

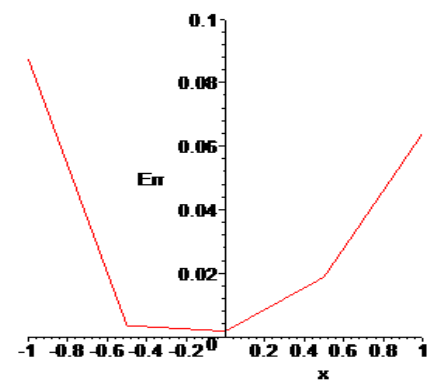

Err. TMM

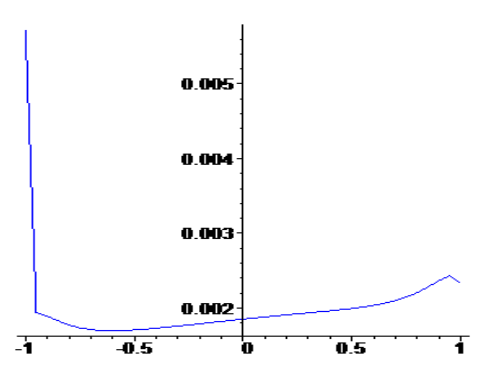

Err. PNM

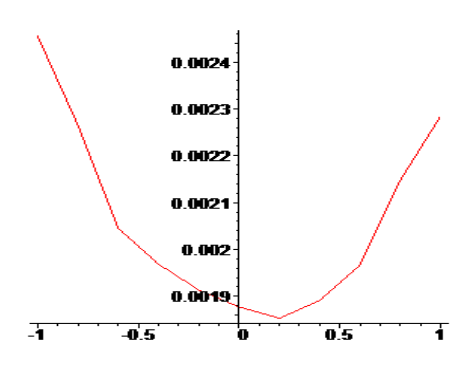

Fig. 9: The error values by using product trapezoidal rule, Toeplitz matrix method and product Nystrom method at $\lambda=0.01$ 
Err. PTR

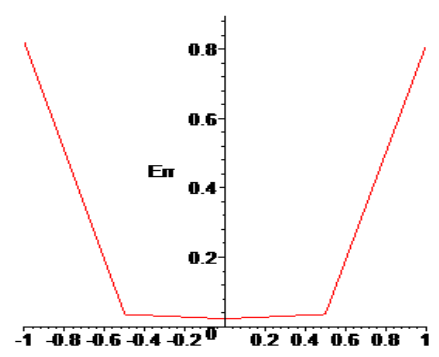

Err. TMM

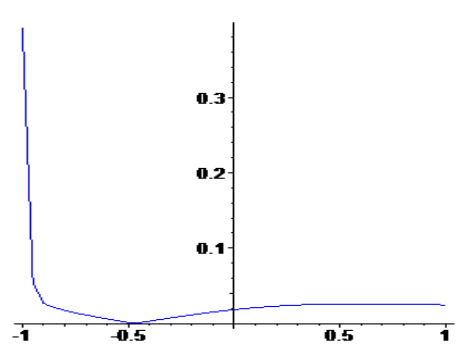

Err. PNM

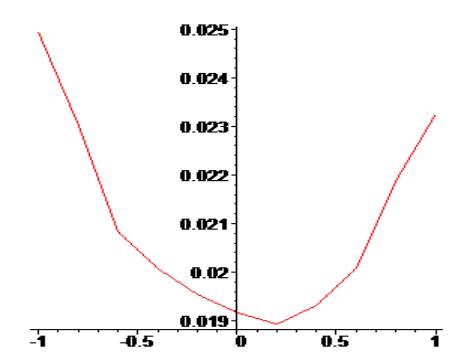

Fig. 10: The error values by using product trapezoidal rule, Toeplitz matrix method and product Nystrom method at $\lambda=0.1$

Example 6: Consider the integral equation

$\phi(t)-\lambda \int_{-1}^{1}\left(\frac{1}{e^{t}-e^{\zeta}}\right) \phi^{2}(\zeta) d \zeta=f(t), \quad\left(\right.$ exact solution $\left.\phi(t)=e^{t}\right)$

Here, the Toeplitz matrix method and product Nystrom method are used to get approximate solution for values of $\mu=1$, $\lambda=0.001,0.01$ and $N=41$ units

Err. PTR

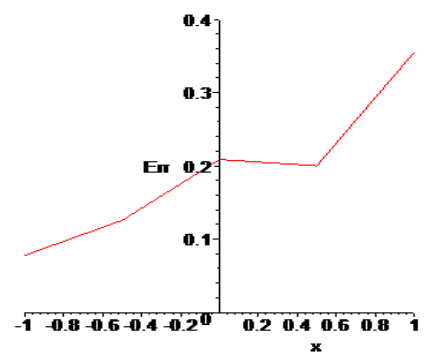

Err. TMM

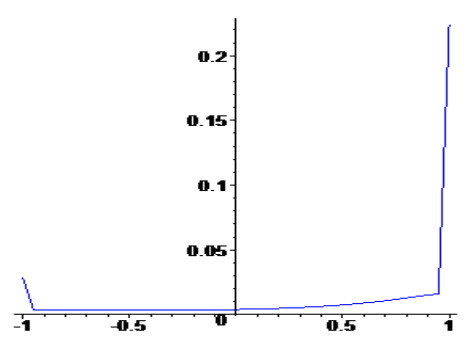

Err. PNM

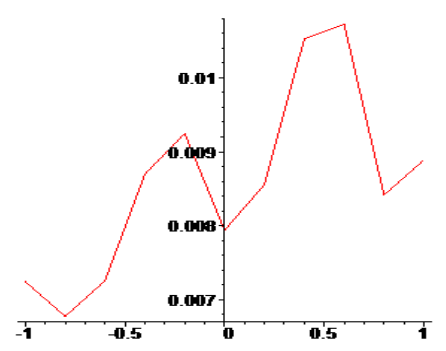

Fig. 11: The error values by using product trapezoidal rule, Toeplitz matrix method and product Nystrom method at $\lambda=0.001$

\section{Err. PTR}

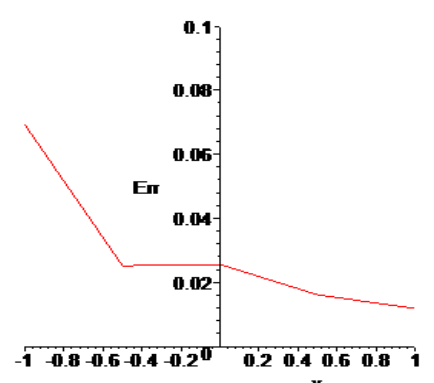

Err. TMM

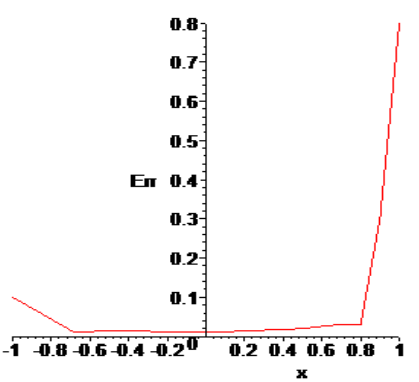

Err. PNM

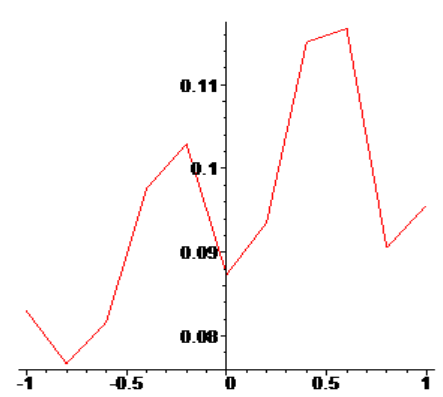

Fig. 12: The error values by using product trapezoidal rule, Toeplitz matrix method and product Nystrom method at $\lambda=0.01$ 


\section{Conclusions}

From the above examples, we deduce the following:

1. When the values of $\lambda$ are increasing, the error is increasing.

2. The error increase when the values of $N$ and $\lambda$ are fixed and when values of $v$ increasing where $v$, in the theory of elasticity is called Poisson's ratio.

3. For all cases we have studied, the product trapezoidal rule and the Toeplitz matrix method are easily programming than product Nystrom method.

\section{References}

[1] N.I .Muskhelishvili, Singular Integral Equations, Noordhoff, Groningen.The Netherlands,1953.

[2] M.A. Abdou, K.I. Mohamed and A.S. Ismail, Toeplitz matrix and product Nystrom methods for solving the singular integral equation, Le Mathematical, vol. Lv11 (2002),Fasc 2.pp.21-37.

[3] M.A. Abdou, M.M. EL-Boraie, M.K. EL.Kojok, Toeplitz matrix method for solving the nonlinear integral equation of Hammerstein type, J. Comp.Appl.Math.223 (2009)765-776.

[4] M. A. Abdou, F. A. Hendi, The numerical solution of Fredholm integral equation with Hilbert kernel, JKSIAM. 9(1) (2005) 111-123.

[5] M. A. Abdou, On the solution of linear and nonlinear integral equation, Appl. Math. Comput, 146 (2003) 857-871.

[6] M.A.Abdou, A.M.Albugami, Numerical Solution for Fredholm Integral Equation with a Generalized Singular Kernel, International Journal of Computational and Applied Mathematics, ISSN 1819-4966 Vol. 7, No. 4 (2012), pp. 449-463.

[7] M. Jafari Emamzadeh, M. Tavassoli Kajani, Nonlinear Fredholm Integral Equation of the Second Kind with Quadrature Methods, Journal of Mathematical Extension, Vol. 4, No. 2 (2010), 51-58.

[8] Ahmad Shahsavaran, Akbar Shahsavaran, Numerical Approach to Solve Second Kind Nonlinear Integral Equations Using Lagrange Functions, Applied Mathematical Sciences, Vol. 6, 2012, no. 18, 893 - 899.

[9] E. Kreysig, Introductory Functional Analysis with Applications, John Wiley \& Sons, New York, 1978.

[10] L.M. Delves and J.L. Mohamed, Computational Methods for Integral Equations, University press, 1985. 